\title{
Associations between estimated and measured carotid-femoral pulse wave velocity in older Black and White adults: the atherosclerosis risk in communities (ARIC) study
}

\author{
Kevin Heffernan ${ }^{1}$, Lee Stoner ${ }^{2}$, Michelle L. Meyer ${ }^{3}$, Adam Keifer ${ }^{2}$, Lauren Bates ${ }^{2}$, Patricia Pagan Lassalle², \\ Erik D. Hanson ${ }^{2}$, Masahiro Horiuchi ${ }^{4}$, Erin D. Michos ${ }^{5}$, Anna Kucharska-Newton ${ }^{6,7}$, Kunihiro Matsushita ${ }^{8}$, \\ Timothy M. Hughes ${ }^{9}$, Hirofumi Tanaka ${ }^{10}$ \\ 'Department of Exercise Science, Syracuse University, Syracuse, NY 13244, USA. \\ ${ }^{2}$ Department of Exercise and Sport Science, University of North Carolina at Chapel Hill, Chapel Hill, NC 27599, USA. \\ ${ }^{3}$ Department of Emergency Medicine, School of Medicine, University of North Carolina at Chapel Hill, Chapel Hill, NC 27599, \\ USA. \\ ${ }^{4}$ Department of Human Environmental Science, Mount Fuji Research Institute, Yamanashi 403-0005, Japan. \\ ${ }^{5}$ Division of Cardiology, Johns Hopkins University School of Medicine, Baltimore, MD 21205, USA. \\ ${ }^{6}$ Department of Epidemiology, The Gillings School of Global Public Health, University of North Carolina at Chapel Hill, Chapel \\ Hill, NC 27514, USA. \\ ${ }^{7}$ Department of Epidemiology, College of Public Health, University of Kentucky, Lexington, KY 40536, USA. \\ ${ }^{8}$ Department of Epidemiology, Johns Hopkins Bloomberg School of Public Health, Baltimore, MD 21205, USA. \\ ${ }^{9}$ Section of Gerontology and Geriatric Medicine, Department of Internal Medicine, Wake Forest School of Medicine, Winston \\ Salem, NC 27157, USA. \\ ${ }^{10}$ Department of Kinesiology and Health Education, The University of Texas at Austin, Austin, TX 78712, USA.
}

Correspondence to: Dr. Lee Stoner, Interdisciplinary Stem Cell Institute, Department of Exercise and Sport Science, University of North Carolina at Chapel Hill, 210 South Road, Chapel Hill, NC 27599, USA. E-mail: dr.I.stoner@gmail.com

How to cite this article: Heffernan K, Stoner L, Meyer ML, Keifer A, Bates L, Lassalle PP, Hanson ED, Horiuchi M, Michos ED, Kucharska-Newton A, Matsushita K, Hughes TM, Tanaka H. Associations between estimated and measured carotid-femoral pulse wave velocity in older Black and White adults: the atherosclerosis risk in communities (ARIC) study. J Cardiovasc Aging 2022;2:7. https://dx.doi.org/10.20517/jca.2021.22

Received: 15 Sep 2021 First decision: 28 Sep 2021 Revised: 10 Nov 2021 Accepted: 18 Nov 2021 Published: 4 Jan 2022

Academic Editor: AJ Marian Copy Editor: Xi-Jun Chen Production Editor: Xi-Jun Chen

\begin{abstract}
Introduction: Aortic stiffness offers important insight into vascular aging and cardiovascular disease (CVD) risk. The referent measure of aortic stiffness is carotid-femoral pulse wave velocity (cfPWV). cfPWV can be estimated (ePWV) from age and mean arterial pressure. Few studies have directly compared the association of ePWV to measured cfPWV, particularly in non-White adults. Moreover, whether ePWV and cfPWV correlate similarly with
\end{abstract}


CVD risk remains unexplored.

Aim: (1) To estimate the strength of the agreement between ePWV and cfPWV in both Black and White older adults; and (2) to compare the associations of ePWV and cfPWV with CVD risk factors and determine whether these associations were consistent across races.

Methods and Results: We evaluated 4478 [75.2 (SD 5.0) years] Black and White older adults in the Atherosclerosis Risk in Communities (ARIC) Study. cfPWV was measured using an automated pulse waveform analyzer. ePWV was derived from an equation based on age and mean arterial pressure. Association and agreement between the two measurements were determined using Pearson's correlation coefficient ( $r$ ), standard error of estimate (SEE), and Bland-Altman analysis. Associations between traditional risk factors with ePWV and cfPWV were evaluated using linear mixed regression models. We observed weak correlations between ePWV and cfPWV within White adults $(r=0.36)$ and Black adults $(r=0.31)$. The mean bias for Bland-Altman analysis was low at $-0.17 \mathrm{~m} / \mathrm{s}(95 \% \mathrm{Cl}$ : -0.25 to -0.09$)$. However, the inspection of the Bland-Altman plots indicated systematic bias $(P<0.001)$, which was consistent across race strata. The SEE, or typical absolute error, was $2.8 \mathrm{~m} / \mathrm{s}$ suggesting high variability across measures. In models adjusted for sex, prevalent diabetes, the number of prevalent cardiovascular diseases, and medication count, both cfPWV and ePWV were positively associated with heart rate, triglycerides, and fasting glucose, and negatively associated with body mass index (BMI) and smoking status in White adults $(P<0.05)$. cfPWV and ePWV were not associated with heart rate, triglycerides, and fasting glucose in Black adults, while both measures were negatively associated with BMI in Black adults.

Conclusions: Findings suggest a weak association between ePWV and cfPWV in older White and Black adults from ARIC. There were similar weak associations between CVD risk factors with ePWV and cfPWV in White adults with subtle differences in associations in Black adults.

One sentence summary: Estimated pulse wave velocity is weakly associated with measured carotid-femoral pulse wave velocity in older Black and White adults in ARIC.

Keywords: Vascular stiffness, measurement, health disparities, pulse wave velocity, blood pressure

\section{INTRODUCTION}

Carotid-femoral pulse wave velocity (cfPWV) is the referent measure of aortic stiffness ${ }^{[1]}$, a pre-clinical measure of vascular aging associated with the development of hypertension, and a predictor of target organ damage $^{[2,3]}$ and future cardiovascular disease (CVD) events ${ }^{[4,5]}$. cfPWV is a fairly well-standardized technique but requires technical proficiency and somewhat expensive equipment that limits its broader application to clinical use. A simpler approach to estimating aortic stiffness would increase the likelihood of integrating this measurement into clinical practice.

A number of technical and procedural modifications, including the use of a thigh cuff, have been attempted to simplify cfPWV ${ }^{[6]}$. Additionally, cfPWV can be estimated (ePWV) using only age and mean arterial pressure (MAP) ${ }^{[7-11]}$. In select cohorts, the relationship between ePWV and cfPWV has been shown to be moderately high $(r=0.52-0.67)^{[12]}$, with emerging studies noting ePWV as a significant predictor of CVD events and all-cause mortality (including following adjustment for age and blood pressure ${ }^{[8,11-14]}$. The original equation to derive ePWV was developed from European cohort data and included predominantly White participants ${ }^{[7]}$. Therefore, while ePWV is potentially a simple and useful tool for predicting CVD risk, further work is warranted to compare ePWV to cfPWV as a measure of arterial stiffness in non-White populations. 
The primary aim of the current study was to determine the strength of the association and level of agreement between ePWV and cfPWV and determine whether association and agreement were consistent across races. The second aim was to compare the strength of associations of ePWV and cfPWV with traditional CVD risk factors and determine whether these associations were consistent across races. These aims were tested using a well-characterized population of older Black and White adults from the Atherosclerosis Risk in Communities (ARIC) Study cohort.

\section{METHODS}

This observational study is reported in accordance with STROBE (STrengthening the Reporting of OBservational studies in Epidemiology) guidelines ${ }^{[15]}$. Participants provided written informed consent, and the study was approved by the Institutional Review Boards at all field centers, coordinating center, and central labs and reading centers. Data availability and detailed policies for requesting ARIC data can be found at https://sites.cscc.unc.edu/aric/pubs-policies-and-forms-pg. Select ARIC data can also be obtained from the NHLBI BioLINCC repository (https://biolincc.nhlbi.nih.gov/home/).

\section{Participants}

The ARIC Study is a population-based, longitudinal study of 15,792 participants aged 45-64 years enrolled between 1987 and 1989 from 4 United States communities (Forsyth County, North Carolina; Jackson, Mississippi; Minneapolis, Minnesota; and Washington County, Maryland). Details of the baseline visit have been previously described ${ }^{[16]}$. The study sample included 5683 participants who had cfPWV measured at visit 5 between 2011 and 2013 .

We excluded participants with the following pre-existing conditions due to concerns over the quality of the PWV measures: BMI $\geq 40 \mathrm{~kg} / \mathrm{m}^{2}$, major arrhythmias (Minnesota codes 8-1-3, 8-3-1, and 8-3-2), Minnesota code 8-1-2 with evidence of biased PWV waveforms, aortic aneurysms, abdominal aorta $\geq 5 \mathrm{~cm}$, history of aortic or peripheral revascularization or aortic graft, aortic stenosis, and moderate or greater aortic regurgitation. Additionally, we excluded participants whose race was other than White or Black (due to small sample size), with missing PWV or vascular risk factor data, as well as those with outlying PWV values, defined as PWV values $>3$ standard deviations above or below the mean. Thus, of the original 5683 participants, 1205 were excluded because they had one or more of the exclusion conditions: pre-existing conditions $(n=579)$, missing cfPWV data $(n=433)$, cfPWV values 3 SDs away from the mean $(n=33)$, race other than White or Black $(n=13)$, missing risk factor data $(n=135)$, and missing covariates $(n=12)$.

\section{Study design}

Participants were asked not to consume food or drink, and refrain from tobacco and vigorous physical activity after midnight prior to the clinic visit or $8 \mathrm{~h}$ prior to the visit. Visit 5 study examination included interviewer-administered questionnaires to obtain demographic data, medical history and lifestyle information, blood and urine collection, and assessment of vascular risk factors and cardiovascular phenotypes, including PWV.

\section{Outcome measures}

\section{Carotid-Femoral pulse wave velocity}

Following 5-10 min of supine rest, technicians measured cfPWV following a standardized protocol with the automated pulse waveform analyzer VP-1000 Plus (Omron, Kyoto, Japan) $)^{[17]}$. cfPWV was estimated as the distance between two arterial recording sites divided by transit time. The distance from the carotid to the femoral artery was directly measured with a segmometer (Rosscraft, Surrey, Canada) and calculated as the carotid to femoral distance minus the distance between the suprasternal notch and the carotid applanation site. To calculate transit time, arterial waveforms were simultaneously acquired for $30 \mathrm{~s}$ by applanation 
tonometry sensors placed on the left common carotid artery (via neck collar) and left common femoral artery. A minimum of two measurements was taken per participant, and the last two measurements were averaged. The validity and reliability of the automatic device for measuring PWV have been described previously ${ }^{[18]}$. Quality assurance for PWV included central training and recertification, quarterly equipment calibration, and ongoing quality control reviews by one of the authors (Tanaka $\mathrm{H}$ ) on a stratified random sample of 40 records per month, with feedback provided to technicians. Approximately $78 \%$ of records were considered optimal quality, $17 \%$ were good quality, $3 \%$ were acceptable, and none were poor or unacceptable.

\section{Estimated pulse wave velocity}

Three seated blood pressure (BP) measurements were obtained after a 5 min rest using an oscillometric automated sphygmomanometer (Omron HEM-907 XL, Omron, Kyoto, Japan), and the average of the last 2 measurements was used. ePWV was calculated using equations published by the Reference Values for Arterial Stiffness Collaboration ${ }^{[7]}$, which use age and MAP and take nonlinearity and interactions into account.

$\mathrm{ePWV}=$

(1) Version $1(V 1) 7.84-0.33 \times$ age $+3.8 \times 10^{-3} \times a^{2 g e^{2}}-1.97 \times 10^{-5} \times \operatorname{age}^{2} \times M A P+2.5 \times 10^{-3} \times($ age $\times M A P)-$ $1.9 \times 10^{-3} \times M A P$

(2) Version $2(V 2) 9.587-0.402 \times$ age $+4.560 \times 10^{-3} \times a^{2 g e^{2}}-2.621 \times 10^{-5} \times \operatorname{age}^{2} \times M A P+3.176 \times 10^{-3} \times$ age $\times$ $M A P-1.832 \times 10^{-2} \times M A P$

Mean arterial pressure was calculated from systolic blood pressure (SBP) and diastolic blood pressure (DBP) as follows: DBP + 0.4 (SBP - DBP), where SBP - DBP is pulse pressure. We chose a form factor of 0.4 for the calculation of MAP in agreement with that used by the Reference Values for Arterial Stiffness Collaboration $^{[7]}$. There is currently no consensus on the optimal formula to estimate MAP from SBP and DBP. A traditional 0.33 form factor may be more appropriate for younger adults with higher SBP amplification, while a form factor 0.4 may be more optimal for those with lower SBP amplification (i.e., older adults) ${ }^{[19]}$. It should be noted that MAP calculated from a form factor of 0.41 has previously been shown to be more closely associated with target organ damage than a traditional form factor of $0.33^{[20]}$. Equation 1 (V1), as used by Vishram-Nielsen et al. ${ }^{[13]}$ in the MOnica Risk, Genetics, Archiving, and Monograph Prospective Cohort Project, utilizes a regression equation derived from a reference population with low-moderate CVD risk. We also explored a second regression equation used by Greve et al. ${ }^{[12]}$ that was derived from a cohort with higher CVD risk. This particular equation was shown to predict survival in the Systolic Blood Pressure Intervention Trial (SPRINT) study involving hypertensive patients ${ }^{[14]}$.

\section{Predictor variables: traditional cvd risk factors}

For aim 2, the cross-sectional associations between ePWV and cfPWV with the following traditional risk factors were explored: BMI, current smoking, heart rate, blood glucose, triglycerides, high-density lipoprotein (HDL) cholesterol, and low-density lipoprotein (LDL) cholesterol. Although heart rate is not a traditional CVD risk factor, it has been shown to be an important correlate of $\mathrm{cfPWV}^{[21,22]}$. Body weight was measured to the nearest $0.1 \mathrm{~kg}$, and height was recorded to the nearest centimeter. BMI was calculated using height and weight. The history of smoking was self-reported and analyzed as dichotomous (current $v s$. noncurrent). Blood samples were obtained following a standardized venipuncture protocol and shipped weekly to ARIC central laboratories, where assays for total cholesterol, HDL cholesterol, triglycerides, and fasting glucose concentration were performed. Total plasma cholesterol concentrations were determined enzymatically ${ }^{[23]}$ using a Cobas-Bio analyzer with reagents purchased from Boehringer Mannheim 
Biochemicals, (Indianapolis, IN). Plasma LDL cholesterol, concentration was calculated using the Friedewald equation ${ }^{[24]}$, and HDL concentrations were measured using the method of Warnick et al. ${ }^{[25]}$.

\section{Covariate measurements}

Age was calculated from the date of birth. Sex and race were self-reported. Participants were asked to bring all prescription and nonprescription medications taken within 2 weeks. That information was transcribed and categorized using MediSPAN prescription codes and classified into medication categories. Participants also self-reported medication use. Medications used included $\beta$-blockers, $\alpha$-blockers, calcium channel blockers, diuretics, angiotensin-converting enzyme inhibitors, and angiotensin II receptor blockers. Diabetes was defined as fasting glucose $\geq 126 \mathrm{mg} / \mathrm{dL}$, non-fasting glucose $\geq 200 \mathrm{mg} / \mathrm{dL}$, anti-diabetic medication use, or self-reported diagnosis of diabetes. Hypertension was defined as SBP $\geq 140 \mathrm{mmHg}$ or $\mathrm{DBP} \geq 90 \mathrm{mmHg}$, or antihypertensive medication use. Prevalent coronary heart disease and stroke were defined by ARIC cohort surveillance data at Visit 5. Prevalent heart failure was defined as physicianreported heart failure or a hospitalization discharge with an ICD code 428.x.

\section{Statistical analyses}

Statistical analyses were performed using R Statistical Software. The $\alpha$-level was set a priori for all statistical procedures at 0.05. Cumulative frequency and Q-Q plots were used to compare the distributions of cfPWV and ePWV. Participant characteristics were stratified by race and estimated as means and SDs, or frequencies and percent, where appropriate. Race differences in demographic characteristics were assessed using chi-squared tests for categorical variables and independent $t$-tests for continuous variables.

For aim 1, we used linear regression to test whether race moderates the relationship between ePWV and cfPWV. Nonlinearity was explored by specifying the ePWV quadratic term. Subsequently, the correlation between the two measurements was determined by calculating the Pearson product-moment correlation $(r)$ and standard error of estimate (SEE). Although there is no universal criterion, in general, $r$ value estimates of $<0.2,0.2-0.4,0.4-0.70,0.70-0.9$ and $>0.9$ indicate negligible, weak, moderate, strong, and very strong correlation, respectively ${ }^{[26]}$. The SEE represents the average distance that the observed values fall from the regression line, with smaller values indicating that the observations are closer to the fitted line. The SEE was calculated using the equation:

$$
S D \times \sqrt{\left(1-r^{2}\right)}
$$

whereby $S D$ is the standard deviation of the criterion measure and $\mathrm{r}$ is the Pearson product-moment correlation between test and criterion devices. The relative standard error was also calculated by expressing SEE relative to the mean of cfPWV. Bland-Altman plots were generated to permit visual analysis of the uniformity of error over the range of participant measurement values ${ }^{[27]}$.

For aim 2, associations between traditional risk factors with ePWV and cfPWV were evaluated using linear mixed regression models with field center specified as a random intercept. Independent variables included BMI, current smoking, heart rate, glucose, HDL cholesterol, LDL cholesterol, and triglycerides. Initially, univariate analysis was conducted, in each traditional risk factor was independently regressed against cfPWV. This analysis was repeated for ePWV. Subsequently, a multivariable analysis was performed, with the traditional risk factors simultaneously regressed against cfPWV. The analysis was repeated for ePWV. These models were then adjusted for sex, prevalent diabetes, number of prevalent cardiovascular diseases (hypertension, coronary heart disease, heart failure, stroke), and medication count. Adjusted variable models were further stratified by race. Assumptions of linearity, collinearity, homoscedasticity, and outliers 
were assessed for each model. We report $\beta$ coefficient estimates, their precision (95\% CI), and the $R^{2}$ values for the models.

\section{RESULTS}

\section{Participants}

Descriptive characteristics are reported in Table 1. Following exclusions, the study sample included 4478 participants between the ages of 66 and 90 years, of which 59\% were women and 23\% were Black. Black participants had higher mean cfPWV $(\Delta=0.9 \mathrm{~m} / \mathrm{s}$, 95\%CI: 0.7-1.1) and ePWV (0.1 m/s, 95\%CI: 0.0-0.2) values compared to Whites, although distributions were similar for each race [Supplementary Figure 1]. Compared to Whites, Black participants had higher (worse) MAP $(P<0.001)$, BMI $(P<0.001)$, heart rate $(P$ $<0.001)$, fasting glucose $(P<0.019)$ and LDL-cholesterol $(P<0.001)$ but had more favorable HDL cholesterol $(P=0.006)$ and triglycerides $(P<0.001)$. Black participants also had a greater proportion of diabetes $(P<0.001)$, hypertension $(P<0.001)$, heart failure $(P<0.001)$ and stroke $(P<0.001)$, and more Black participants used each class of medication (all $P<0.001$ ).

\section{Agreement between ePWV and cfPWV}

Nonlinearity was explored by specifying the ePWV quadratic term for the total population, and in analyses, stratified by race [Supplementary Table 1]. The quadratic term was non-significant for the total sample population, and within the White $(P=0.380)$ and Black strata. Thus, linear models were used for subsequent analyses.

Correlations between ePWV and cfPWV are reported in Table 2. We observed a weak $(r=0.35,95 \% \mathrm{CI}$ : $0.32-0.37)$ correlation between ePWV and cfPWV for the total population, with comparable correlations for White adults ( $r=0.36,95 \% \mathrm{CI}: 0.33-0.39)$ and Black adults $(r=0.31,95 \% \mathrm{CI}: 0.26-0.37)$. Bland-Altman analysis [Figure 1 and Supplementary Table 2] indicated a mean bias of $-0.17 \mathrm{~m} / \mathrm{s}$ (95\%CI: -0.25 to -0.09 ). However, the small mean bias is misleading; inspection of the regression [Figure $1 \mathrm{~A}$ and $\mathrm{B}$ ] and BlandAltman [Figure $1 \mathrm{C}$ and $\mathrm{D}$ ] plots indicated significant $(P<0.001)$ proportional bias, which was consistent across race strata. The SEE, or typical absolute error, was high at $2.8 \mathrm{~m} / \mathrm{s}$ suggesting high variability across measures.

Supplementary Table 3 presents the correlation between ePWV using V2 and cfPWV ( $r=0.35,95 \% \mathrm{CI}$ : 0.320.37), which was comparable to the correlations between ePWV using V1 and cfPWV, including when stratified by race.

\section{Correlations between cfPWV and ePWV with traditional cardiovascular disease risk factors}

Table 3 presents the associations between traditional cardiovascular disease risk factors and cfPWV and ePWV. In adjusted models (sex, prevalent diabetes, number of prevalent cardiovascular diseases, and medication count), cfPWV was positively associated with heart rate, triglycerides, and fasting glucose, and negatively associated with BMI, HDL cholesterol, and smoking status. ePWV was also positively associated with heart rate and triglycerides, and negatively associated with BMI and smoking status. However, ePWV was not associated with HDL cholesterol or fasting glucose. Neither PWV measure was associated with LDL cholesterol levels in adjusted models. Table 4 presents the associations between traditional cardiovascular disease risk factors with cfPWV and ePWV stratified by race. For Whites, the associations were consistent with those reported for the total population. For Black adults, triglycerides and smoking status were not associated with cfPWV. HDL cholesterol was not associated with ePWV for the total or White populations. HDL showed a positive association with ePWV in Black adults. Similarly, across subgroups, cfPWV and ePWV measures were inversely associated with BMI and smoking status, which is unexpected. Table 5 displays the comparison of ePWV to cfPWV using ARTERY Society Guidelines ${ }^{[28]}$. In general, accuracy 
Table 1. Descriptive characteristics of ARIC visit 5 participants, stratified by sex

\begin{tabular}{|c|c|c|c|c|c|c|c|c|}
\hline \multirow{3}{*}{$\begin{array}{l}\text { Continuous variables } \\
\text { Age (years) }\end{array}$} & \multirow{2}{*}{\multicolumn{2}{|c|}{$\frac{\text { Total }(n=4478)}{\text { Mean }(\text { SD) }}$}} & \multirow{2}{*}{\multicolumn{2}{|c|}{$\begin{array}{c}\text { White }(n=3468) \\
\text { Mean (SD) }\end{array}$}} & \multirow{2}{*}{\multicolumn{2}{|c|}{$\begin{array}{c}\text { Black }(n=1010) \\
\text { Mean }(S D)\end{array}$}} & & \multirow{3}{*}{$\begin{array}{c}\boldsymbol{d} \\
0.19\end{array}$} \\
\hline & & & & & & & & \\
\hline & 75.2 & $(5.03)$ & 75.5 & $(5.04)$ & \multicolumn{2}{|c|}{$74.5 \quad(4.94)$} & & \\
\hline $\operatorname{cfPWV}(\mathrm{m} / \mathrm{s})$ & 11.6 & $(3.02)$ & 11.4 & $(2.90)$ & 12.3 & $(3.34)$ & $<0.001$ & 0.29 \\
\hline ePWV V1 (m/s) & 11.4 & $(1.33)$ & 11.4 & $(1.33)$ & 11.5 & $(1.32)$ & 0.004 & 0.10 \\
\hline ePWV V2 (m/s) & 11.8 & $(1.37)$ & 11.8 & $(1.38)$ & 11.9 & $(1.36)$ & 0.021 & 0.08 \\
\hline Systolic blood pressure $(\mathrm{mmHg})$ & 130 & $(17.6)$ & 129 & $(17.3)$ & 134 & $(18.1)$ & $<0.001$ & 0.27 \\
\hline Diastolic blood pressure $(\mathrm{mmHg})$ & 66.1 & $(10.4)$ & 65.1 & $(10.2)$ & 69.4 & $(10.4)$ & $<0.001$ & 0.42 \\
\hline Pulse pressure $(\mathrm{mmHg})$ & 63.9 & $(14.3)$ & 63.8 & $(14.2)$ & 64.3 & $(14.7)$ & 0.392 & 0.03 \\
\hline Mean arterial pressure $(\mathrm{mmHg})$ & 91.6 & $(11.8)$ & 90.6 & $(11.6)$ & 95.1 & $(12.0)$ & $<0.001$ & 0.38 \\
\hline Body mass index $\left(\mathrm{kg} / \mathrm{m}^{2}\right)$ & 27.8 & $(4.47)$ & 27.4 & $(4.31)$ & 29.2 & $(4.31)$ & $<0.001$ & 0.39 \\
\hline Heart rate (bpm) & 64.7 & $(10.7)$ & 64.3 & $(10.5)$ & 66.0 & $(11.4)$ & $<0.001$ & 0.16 \\
\hline Fasting glucose $(\mathrm{mmol} / \mathrm{L})$ & 6.21 & $(1.45)$ & 6.18 & $(1.40)$ & 6.30 & $(1.62)$ & 0.019 & 0.08 \\
\hline LDL Cholesterol (mmol/L) & 2.74 & $(0.89)$ & 2.71 & $(0.89)$ & 2.86 & $(0.89)$ & $<0.001$ & 0.16 \\
\hline HDL Cholesterol (mmol/L) & 1.37 & $(0.36)$ & 1.36 & $(0.37)$ & 1.40 & $(0.35)$ & 0.006 & 0.10 \\
\hline Triglycerides (mmol/L) & 1.39 & $(0.63)$ & 1.44 & $(0.65)$ & 1.20 & $(0.51)$ & $<0.001$ & 0.40 \\
\hline Categorical variables & \multicolumn{2}{|c|}{ No. (\%) } & \multicolumn{2}{|c|}{ No. (\%) } & \multicolumn{2}{|c|}{ No. (\%) } & $P$ & OR \\
\hline Female & 2661 & 59.4 & 1993 & 57.5 & 668 & 66.1 & $<0.001$ & 1.45 \\
\hline Current smoker & 263 & 5.9 & 197 & 5.7 & 61 & 6.0 & 0.31 & 1.16 \\
\hline Prevalent diabetes & 1314 & 29.3 & 909 & 26.2 & 405 & 40.1 & $<0.001$ & 1.88 \\
\hline \multicolumn{9}{|l|}{ Prevalent cardiovascular disease } \\
\hline Hypertension & 3302 & 73.7 & 2336 & 67.4 & 866 & 85.7 & $<0.001$ & 2.93 \\
\hline Coronary heart disease & 618 & 13.8 & 514 & 14.8 & 104 & 10.3 & 0.654 & 0.65 \\
\hline Heart failure & 473 & 10.6 & 300 & 8.7 & 173 & 17.1 & $<0.001$ & 2.18 \\
\hline Stroke & 129 & 2.9 & 77 & 2.2 & 52 & 5.2 & $<0.001$ & 2.39 \\
\hline Total count (median, Q1, Q3) & 1 & 1,1 & 1 & 1,1 & 1 & 1,2 & & \\
\hline \multicolumn{9}{|l|}{ Cholesterol lowering medications } \\
\hline Primary & 2466 & 55.1 & 1943 & 56.0 & 523 & 51.8 & 0.023 & 0.85 \\
\hline Secondary & 2338 & 52.2 & 1711 & 49.3 & 623 & 61.7 & $<0.001$ & 1.71 \\
\hline \multicolumn{9}{|l|}{ Hypertensive medications } \\
\hline$\beta$-Blocker & 1232 & 27.5 & 1017 & 29.3 & 215 & 21.3 & $<0.001$ & 0.67 \\
\hline$\alpha$-Blocker & 143 & 3.2 & 87 & 2.51 & 56 & 5.5 & $<0.001$ & 2.35 \\
\hline Diuretic & 1678 & 37.5 & 1109 & 32.0 & 569 & 56.3 & $<0.001$ & 2.96 \\
\hline ACE inhibitor & 1336 & 29.8 & 986 & 28.4 & 350 & 34.7 & $<0.001$ & 1.39 \\
\hline ANG II receptor blocker & 726 & 16.2 & 492 & 14.2 & 234 & 23.2 & $<0.001$ & 1.89 \\
\hline Calcium channel blocker & 1072 & 23.9 & 674 & 19.4 & 398 & 39.4 & $<0.001$ & 2.84 \\
\hline Total count (median, Q1, Q3) & 1 & 0,2 & 1 & 0,2 & 2 & 1,3 & & \\
\hline
\end{tabular}

cfPWV: Carotid-femoral pulse wave velocity; ePWV: estimated pulse wave velocity; V1: ePWV equation version 1; V2: ePWV equation version 2; LDL: low-density lipoprotein; HDL: high-density lipoprotein; ACE: angiotensin converting enzyme; ANG: angiotensin.

across measures was considered acceptable-excellent (mean difference between measures $<1.0 \mathrm{~m} / \mathrm{s}$ ) in approximately $30 \%$ of ARIC participants.

Supplementary Table 4 presents the associations between traditional cardiovascular disease risk factors with ePWV V2 for the total group and stratified by race. The findings are consistent with those for ePWV V1. Supplementary Table 5 presents the association of age, MAP, and age-MAP interaction term with cfPWV for the total group and stratified by race. 
Table 2. Comparison of estimated pulse-wave velocity (ePWV V1) and carotid-femoral pulse wave velocity (cfPWV), stratified by race

\begin{tabular}{|c|c|c|c|c|c|c|c|c|c|c|c|}
\hline \multirow[b]{3}{*}{ Total } & \multirow{3}{*}{$\begin{array}{c}n \\
4478\end{array}$} & \multirow{2}{*}{\multicolumn{2}{|c|}{$\begin{array}{c}\text { cfPWV } \\
\text { Mean (SD) }\end{array}$}} & \multirow{2}{*}{\multicolumn{2}{|c|}{$\begin{array}{c}\text { ePWV } \\
\text { Mean (SD) }\end{array}$}} & \multirow{2}{*}{\multicolumn{2}{|c|}{$\begin{array}{c}r \\
(95 \% \mathrm{Cl})\end{array}$}} & \multirow{2}{*}{\multicolumn{2}{|c|}{$\begin{array}{c}\text { SEE } \\
(95 \% \mathrm{Cl})\end{array}$}} & \multirow{2}{*}{\multicolumn{2}{|c|}{$\begin{array}{c}\text { RSE } \\
(95 \% \mathrm{CI})\end{array}$}} \\
\hline & & & & & & & & & & & \\
\hline & & 11.6 & (3.0) & 11.4 & (1.3) & 0.35 & $(0.32-0.37)$ & 2.8 & $(2.8-2.9)$ & 24.4 & $(24.2-24.6)$ \\
\hline White & 2489 & 11.4 & (2.9) & 11.4 & (1.3) & 0.36 & $(0.33-0.39)$ & 2.7 & $(2.7-2.7)$ & 23.8 & $(23.5-24.0)$ \\
\hline Black & 1644 & 12.3 & (3.3) & 11.5 & (1.3) & 0.31 & $(0.26-0.37)$ & 3.2 & $(3.1-3.2)$ & 25.8 & $(25.3-26.3)$ \\
\hline
\end{tabular}

$\mathrm{Cl}$ : Confidence interval; SEE: standard error of estimate; $r$ : Pearson's correlation coefficient; RSE: relative standard error.

Table 3. Multivariable associations between estimated pulse-wave velocity (ePWV V1) and carotid-femoral pulse wave velocity (cfPWV) with traditional vascular risk factors

\begin{tabular}{|c|c|c|c|c|c|c|c|c|}
\hline & \multicolumn{4}{|c|}{ Unadjusted $(n=4478)$} & \multicolumn{4}{|c|}{ Adjusted $(n=4478)$} \\
\hline & $\boldsymbol{\beta}$ & SE & Std. $\beta$ & $\boldsymbol{P}$ & $\boldsymbol{\beta}$ & SE & Std. $\beta$ & $\boldsymbol{P}$ \\
\hline cfPWV & & $R^{2}$ & 0.11 & & & $R^{2}$ & 0.11 & \\
\hline Body mass index $\left(\mathrm{kg} / \mathrm{m}^{2}\right)$ & -0.082 & 0.011 & -0.134 & $<0.001$ & -0.088 & 0.011 & -0.130 & $<0.001$ \\
\hline Heart rate (bpm) & 0.053 & 0.004 & 0.171 & $<0.001$ & 0.058 & 0.004 & 0.207 & $<0.001$ \\
\hline $\mathrm{HDL}(\mathrm{mmol} / \mathrm{L})$ & -0.643 & 0.138 & -0.045 & $<0.001$ & -0.300 & 0.149 & -0.036 & 0.044 \\
\hline $\mathrm{LDL}(\mathrm{mmol} / \mathrm{L})$ & -0.117 & 0.052 & -0.003 & 0.023 & -0.002 & 0.053 & 0.000 & 0.974 \\
\hline Triglycerides (mmol/L) & 0.314 & 0.078 & 0.041 & $<0.001$ & 0.337 & 0.078 & 0.070 & $<0.001$ \\
\hline Fasting glucose (mmol/L) & 0.222 & 0.032 & 0.096 & $<0.001$ & 0.178 & 0.032 & 0.086 & $<0.001$ \\
\hline Smoker status (current vs. noncurrent) & -0.362 & 0.184 & -0.015 & 0.050 & -0.392 & 0.185 & -0.030 & 0.034 \\
\hline ePWV & & $R^{2}$ & 0.02 & & & $R^{2}$ & 0.06 & \\
\hline Body mass index $\left(\mathrm{kg} / \mathrm{m}^{2}\right)$ & -0.025 & 0.005 & -0.084 & $<0.001$ & -0.024 & 0.005 & -0.080 & $<0.001$ \\
\hline Heart rate (bpm) & 0.004 & 0.002 & 0.035 & 0.019 & 0.005 & 0.002 & 0.042 & 0.005 \\
\hline $\mathrm{HDL}(\mathrm{mmol} / \mathrm{L})$ & 0.016 & 0.063 & 0.004 & 0.800 & 0.104 & 0.067 & 0.028 & 0.124 \\
\hline LDL (mmol/L) & -0.008 & 0.024 & -0.005 & 0.745 & 0.045 & 0.024 & 0.030 & 0.056 \\
\hline Triglycerides (mmol/L) & 0.090 & 0.035 & 0.042 & 0.012 & 0.070 & 0.035 & 0.033 & 0.047 \\
\hline Fasting glucose (mmol/L) & -0.016 & 0.014 & -0.018 & 0.258 & -0.019 & 0.014 & -0.021 & 0.189 \\
\hline Smoker status (current vs. noncurrent) & -0.529 & 0.084 & -0.093 & $<0.001$ & -0.535 & 0.084 & -0.094 & $<0.001$ \\
\hline
\end{tabular}

Adjustments: sex; prevalent cardiovascular diseases (hypertension, coronary heart disease, stroke, heart failure); medications ( $\beta$-blockers, $\alpha$ blockers, calcium channel, blockers, diuretics). Std. $\beta$ : Standardized beta; SE: standard error; HDL: high-density lipoprotein; LDL: low-density lipoprotein.

\section{DISCUSSION}

This study investigated the association between ePWV and cfPWV in White and Black older adults from ARIC. Our primary finding is that ePWV and cfPWV are weakly correlated in older adults, and these weak associations are similar in older Black and White adults.

\section{Comparison to literature}

To generate the equations for ePWV, the Reference Values for Arterial Stiffness Collaboration culled data from 16,867 participants between the ages of 15-97 years (mean age $50 \pm 17$, equal proportions male and female, 24\% smokers) across 13 different clinical centers spanning 8 different European countries. Participants with type 2 diabetes mellitus, overt CVD and adults being treated for hypertension or dyslipidemia were then excluded resulting in a final study population of 11,092 adults ${ }^{[7]}$. Greve et al. ${ }^{[12]}$ compared ePWV and cfPWV in both the Danish MONItoring of trends and determinants in CArdiovascular disease (MONICA) 10 cohort ( $n=\sim 2300$ individuals from Copenhagen) and the Paris cohort ( $n=\sim 1000$ adults with essential hypertension), reporting a moderate correlation between the two 
Table 4. Multivariable associations between estimated pulse-wave velocity (ePWV V1) and carotid-femoral pulse wave velocity (cfPWV) with traditional vascular risk factors, stratified by race

\begin{tabular}{|c|c|c|c|c|c|c|c|c|}
\hline & \multicolumn{4}{|c|}{$n=3468$} & \multicolumn{4}{|c|}{$n=1010$} \\
\hline & $\boldsymbol{\beta}$ & SE & Std. $\beta$ & $\boldsymbol{P}$ & $\boldsymbol{\beta}$ & SE & Std. $\beta$ & $\mathbf{P}$ \\
\hline cfPWV & & $R^{2}$ & 0.11 & & & $R^{2}$ & 0.13 & \\
\hline Body mass index $\left(\mathrm{kg} / \mathrm{m}^{2}\right)$ & -0.085 & 0.012 & -0.126 & $<0.001$ & -0.109 & 0.024 & -0.153 & $<0.001$ \\
\hline Heart rate (bpm) & 0.062 & 0.005 & 0.223 & $<0.001$ & 0.052 & 0.009 & 0.178 & $<0.001$ \\
\hline $\mathrm{HDL}(\mathrm{mmol} / \mathrm{L})$ & -0.294 & 0.164 & -0.037 & 0.074 & -0.357 & 0.342 & -0.038 & 0.297 \\
\hline $\mathrm{LDL}(\mathrm{mmol} / \mathrm{L})$ & -0.012 & 0.058 & -0.004 & 0.839 & 0.046 & 0.122 & 0.012 & 0.705 \\
\hline Triglycerides (mmol/L) & 0.364 & 0.083 & 0.082 & $<0.001$ & 0.239 & 0.224 & 0.037 & 0.287 \\
\hline Fasting glucose (mmol/L) & 0.181 & 0.036 & 0.088 & $<0.001$ & 0.163 & 0.068 & 0.079 & 0.016 \\
\hline Smoker status (current vs. noncurrent) & -0.401 & 0.204 & -0.032 & 0.049 & -0.388 & 0.427 & -0.028 & 0.364 \\
\hline ePWV & & $R^{2}$ & 0.07 & & & $R^{2}$ & 0.06 & \\
\hline Body mass index $\left(\mathrm{kg} / \mathrm{m}^{2}\right)$ & -0.028 & 0.006 & -0.090 & $<0.001$ & -0.022 & 0.010 & -0.077 & 0.025 \\
\hline Heart rate (bpm) & 0.007 & 0.002 & 0.055 & 0.001 & 0.001 & 0.004 & 0.010 & 0.760 \\
\hline $\mathrm{HDL}(\mathrm{mmol} / \mathrm{L})$ & 0.036 & 0.077 & 0.010 & 0.637 & 0.295 & 0.137 & 0.079 & 0.032 \\
\hline $\mathrm{LDL}(\mathrm{mmol} / \mathrm{L})$ & 0.046 & 0.027 & 0.031 & 0.092 & 0.056 & 0.049 & 0.037 & 0.259 \\
\hline Triglycerides (mmol/L) & 0.105 & 0.039 & 0.051 & 0.007 & -0.078 & 0.090 & -0.030 & 0.384 \\
\hline Fasting glucose (mmol/L) & -0.021 & 0.017 & -0.023 & 0.205 & -0.011 & 0.027 & -0.013 & 0.691 \\
\hline Smoker status (current vs. noncurrent) & -0.530 & 0.096 & -0.092 & $<0.001$ & -0.519 & 0.172 & -0.096 & 0.003 \\
\hline
\end{tabular}

Adjustments: sex; prevalent cardiovascular diseases (hypertension, coronary heart disease, stroke, heart failure); medications ( $\beta$-blockers, $\alpha$ blockers, calcium channel, blockers, diuretics). Std. $\beta$ : Standardized beta; SE: standard error; HDL: high-density lipoprotein; LDL: low-density lipoprotein.

Table 5. Classification of ePWV V1 based on mean differences with cfPWV using ARTERY Society recommendations for comparing the accuracy of devices that measure cfPWV

\begin{tabular}{cccc}
\hline \multicolumn{2}{c}{ Proportion } & Mean difference & Accuracy \\
\hline White $\boldsymbol{n}(\%)$ & Black $\boldsymbol{n}(\%)$ & cfPWV vs. ePWV & Classification \\
\hline $580(17)$ & $138(16)$ & $<0.5 \mathrm{~m} / \mathrm{s}$ & Excellent \\
$515(15)$ & $157(15)$ & $0.5-1.0 \mathrm{~m} / \mathrm{s}$ & Acceptable \\
$2373(71)$ & $715(66)$ & $>1.0 \mathrm{~m} / \mathrm{s}$ & Poor \\
\hline
\end{tabular}

ePWV: Estimated pulse wave velocity; cfPWV: carotid-femoral pulse wave velocity.

measures ( $r$ range: 0.52 to 0.67 ). Hametner et al. ${ }^{[29]}$ compared ePWV to invasively measured aortic PWV measured during cardiac catheterization and noted a similar correlation between ePWV and aortic PWV ( $r$ $=0.67$ ). Association between ePWV and cfPWV was assessed in a subset from this study, and correlations were reported to be slightly lower $(r=0.54)^{[29]}$. Stamatelopoulos et al. ${ }^{[30]}$ recently compared ePWV to cfPWV in 934 adults from the Athens Vascular Registry (mean age 60 years) and noted a correlation of $r=0.64$. Correlations seen herein in ARIC were appreciably lower than previously reported. The original equation to derive ePWV was developed from European cohort data, and researchers stated that subjects other than Caucasians were a small minority in this cohort ${ }^{[7]}$. Therefore, we had originally hypothesized that racial and ethnic variation in arterial stiffness might impact the correlation between ePWV and cfPWV. Black individuals experience hastened rates of vascular aging ${ }^{[1,32]}$. For a given level of BP, Black individuals have stiffer central arteries at every age ${ }^{[33]}$. However, when exploring associations across race in the ARIC cohort, similar weak associations were noted in White and Black adults, suggesting a general lower association between measures irrespective of racial variation. Thus, racial variation may not be a reason for discrepancies in the association between ePWV and cfPWV when comparing findings herein to previous findings ${ }^{[12]}$. 

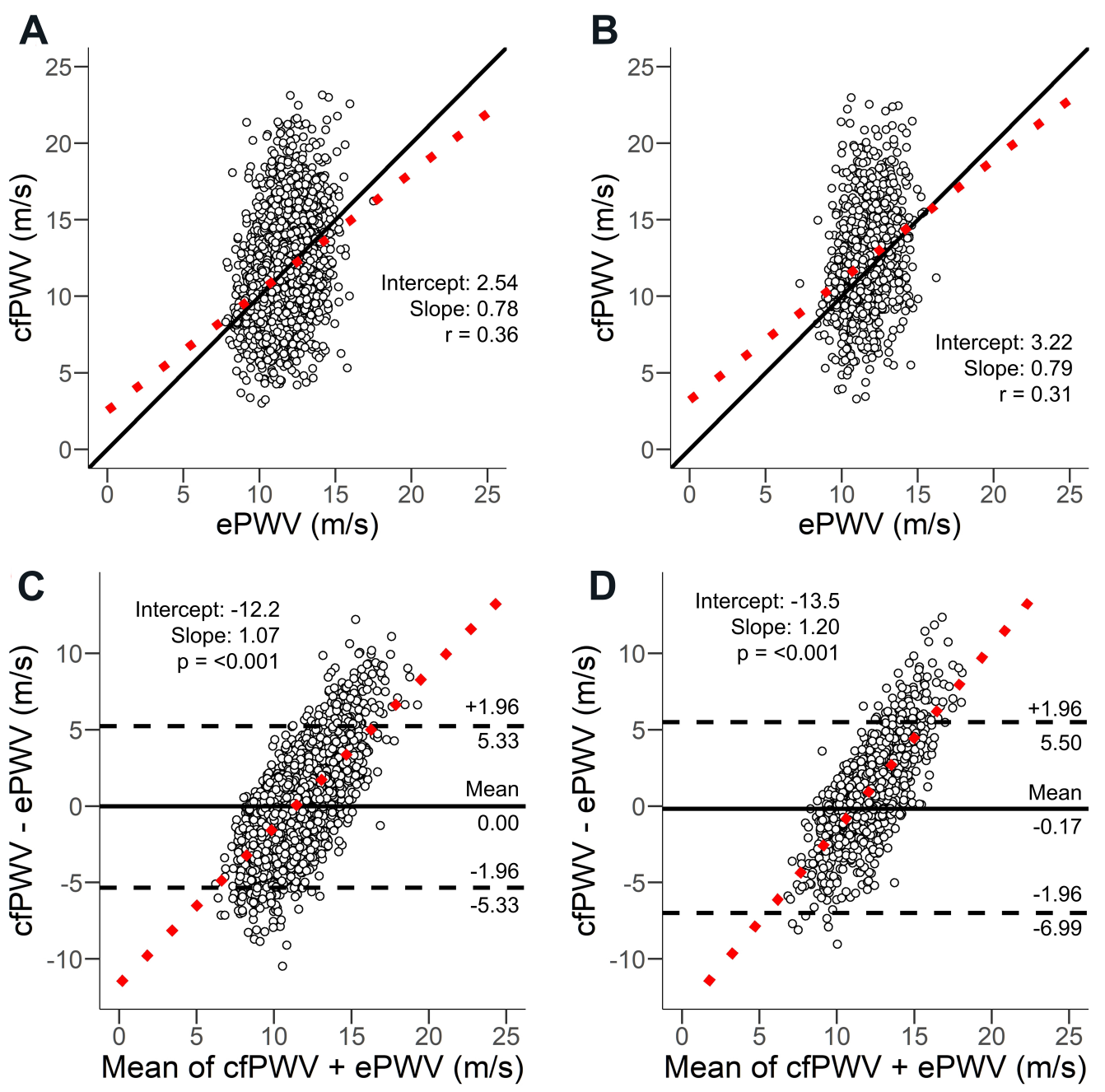

Figure 1. Regression for White adults (A) and Black adults (B). Bland-Altman plots for estimated pulse velocity (ePWV V1) vs. carotidfemoral pulse wave velocity (cfPWV) for White adults (C) and Black adults (D). cfPWV: Carotid-femoral pulse wave velocity; ePWV: estimated pulse wave velocity; V1: ePWV equation version 1; V2: ePWV equation version 2.

As the initial equations generated by the Reference Values for Arterial Stiffness Collaboration excluded participants with overt CVD and adults being treated for hypertension or dyslipidemia, a potential reason for the weak association between ePWV and cfPWV reported herein may be due to the CVD risk status of participants. As can be seen from our data, nearly 30\% of participants in ARIC were diabetic, over $70 \%$ were hypertensive, and an additional $20 \%$ had a history of coronary artery disease, heart failure, or stroke. Hypertension and diabetes both accelerate arterial stiffening with aging ${ }^{[34]}$. Moreover, medications to treat hypertension, diabetes, and dyslipidemia may all have variable effects on blood pressure and its relationship to arterial stiffness. For example, antihypertensive therapy can lower brachial BP but have negligible effects on aortic stiffness ${ }^{[35]}$. Thus, the relationship between blood pressure and cfPWV may be different across CVD status and subsequent medical management, affecting the association between ePWV and cfPWV. The Reference Values for Arterial Stiffness Collaboration study population was also significantly younger than ARIC participants (mean age 55 years vs. 75 years). Thus, discrepancies between ePWV and cfPWV noted herein are likely related to the older age, and higher CVD risk burden of the ARIC cohort compared to other populations studied. 
Other reasons for discrepancy across ePWV and cfPWV may be methodological. The method used by ARIC to estimate path length for cfPWV (i.e., carotid-sternal notch distance subtracted from the carotid to femoral distance) differed from the method used by The Reference Values for Arterial Stiffness Collaboration to standardize cfPWV across study sites (i.e., $80 \%$ of the direct carotid-femoral path length) ${ }^{[7]}$. Differences between path length measurements can result in differences in PWV values by upwards of $30 \%{ }^{[36]}$. Additionally, different methods used to identify the foot of the pressure waveform (i.e., intersecting tangents $v s$. maximal upstroke) may result in PWV differences of $5 \%-15 \%{ }^{[7]}$. Finally, blood pressure and measures of PWV were not performed simultaneously or in the same position (seated $v s$. supine). $\mathrm{BP}$ is higher in the seated position, and given the nature of the equations used for ePWV, a difference in MAP of 3-5 mmHg in a 75-year-old can result in a 0.3-0.4 m/s difference in estimated PWV.

This study further examined the association of traditional CVD risk factors with both measures of PWV. We noted heterogeneity in associations between ePWV and cfPWV with CVD risk factors in analyses stratified by race. In general, arterial stiffness is thought to capture the process of arteriosclerosis, an outside-in process related to structural components of the vessel wall ${ }^{[37]}$. Though sharing pathways in common with atherosclerosis (an inside-out process related to lipid accumulation within the vessel wall), arteriosclerosis has distinct pathophysiology ${ }^{[37]}$. As such, traditional atherosclerotic CVD risk factors (with the exception of age and blood pressure) do not always strongly associate with arterial stiffness ${ }^{[38]}$. Thus, similar to cfPWV, ePWV did not correlate well with many traditional atherosclerotic CVD risk factors. In models adjusted for sex, prevalent diabetes, the number of prevalent cardiovascular diseases, and medication count, both cfPWV and ePWV were positively associated with heart rate and triglycerides and negatively associated with BMI and smoking status. Elevated triglycerides may hasten vascular stiffening via detrimental effects on inflammation and endothelial function ${ }^{[39]}$. Heart rate is known to correlate with arterial stiffness ${ }^{[40]}$. Increased heart rate may expose the vessel wall to more cyclic stress and, when combined with higher blood pressure, may hasten fatigue failure and elastin fracture. The finding of a negative association between BMI and PWV across races in our study, although paradoxical, is consistent with previous findings ${ }^{[41,42]}$. In addition to the explanations previously offered in the literature (e.g., the effect of obesity on stroke volume or reverse epidemiology ${ }^{[41]}$, it is possible that a strong association between path length and height results in collinearity between cfPWV and BMI, altering the directionality of the association. There were also paradoxical negative associations between current smoking status and PWV measures. More research will be needed to explore this smokers paradox.

\section{Implications and additional considerations}

Despite the weak associations between ePWV and cfPWV noted herein, ePWV may still hold promise as a measure of vascular aging and CVD risk. ePWV has been shown to predict cardiovascular and cerebrovascular events and all-cause mortality independent of traditional CVD risk factors (including the following adjustment for age and $\mathrm{BP})^{[8-14,29,43-45]}$. Recently, ePWV was shown to improve risk stratification for all-cause mortality in hospitalized patients with COVID-19 beyond traditional risk factors and risk scores $^{[30]}$. Additionally, reduction of ePWV with antihypertensive treatment in SPRINT predicted survival independent of effects on $\mathrm{BP}^{[14]}$. ePWV has been shown to improve the $\mathrm{C}$-index or net-reclassification index when added to conventional CVD risk scores and improves the area under the receiver-operator characteristic curve beyond traditional CVD risk scores, although this is not a universal finding ${ }^{[1,13,46]}$. Interestingly, Greve et al. ${ }^{[12]}$ initially demonstrated that ePWV retained independent predictive value when added to models with cfPWV and even improved prediction of CVD events beyond cfPWV. Thus, ePWV may be capturing interactions between age and MAP that reflect other aspects of vascular aging that are only partially captured by cfPWV itself ${ }^{[47]}$. 
According to our results [Supplementary Table 5], the correlation between ePWV and cfPWV is similar to that of age + MAP and cfPWV. It is not uncommon for CVD risk factor interactions to predict outcomes more strongly than their constituent components. For example, the interaction of heart rate and systolic blood pressure (i.e., the double product) predicts cardiovascular outcomes and mortality more strongly than heart rate or systolic blood pressure alone and improves prediction when added to heart rate or systolic blood pressure ${ }^{[48,49]}$. As cfPWV is an estimate of aortic stiffness itself that can be influenced by factors other than age and BP (i.e., tortuosity affecting path length), future studies that estimate PWV from cardiac magnetic resonance imaging-based measures of aortic stiffness may also prove valuable. Indeed, ePWV has been proposed as a potentially useful screening tool and "gatekeeper" to help inform additional testing of aortic stiffness with magnetic resonance imaging ${ }^{[50]}$.

\section{Limitations and strengths}

The strengths and limitations of this study need to be addressed to best contextualize the findings. This was a cross-sectional study (non-experimental study design), and our population consisted of older adults with a relatively high CVD risk factor burden, limiting the generalizability of our findings to other populations. While ARIC participants were originally enrolled between 1987 and 1989, cfPWV measures were performed more recently during visit 5 (2011-2013). Thus, our participant population herein is not representative of the general ARIC study cohort at baseline, owing to participant mortality.

Survival bias should be considered when interpreting findings. We excluded participants with BMI > $40 \mathrm{~kg} / \mathrm{m}^{2}$ and cardiac arrhythmias owing to effects of obesity on pressure waveform quality and concerns with arrhythmias in calculating cfPWV. Therefore, our findings extend to older adults that are not Class 3 obese or have cardiac electrophysiological abnormalities. Additionally, since Black participants in the ARIC cohort predominantly reside in Jackson, MS, the observed associations may not generalize to Black Americans as a demographic group. The study population may be biased through the inclusion of participants who have survived from baseline (1987-1989) to the time of the Visit 5 examination (20112013) and are relatively healthier as compared to those who did not participate in the visit. As with any observational study, we cannot rule out the possibility of residual confounding - although we did include several important confounders in our models. A major strength of this study is that it is the largest study to directly compare ePWV and cfPWV assessments in both White and non-White individuals. Finally, it should be underscored that we are not proposing ePWV as a surrogate for or replacement of cfPWV, but rather as a tool to increase awareness on the importance of vascular aging as it relates to CVD risk and as a possible screening aid to help inform additional vascular aging testing with cfPWV or an equivalent method as needed.

\section{Conclusions}

In conclusion, ePWV is weakly associated with cfPWV in older White and Black adults from the ARIC study.

\section{DECLARATIONS}

\section{Authors' contributions}

Conceptualization, helped conceive/design analyses, wrote original draft preparation, wrote review and edited: Heffernan K, Stoner L, Meyer ML

Performed and interpreted analyses, visualization: Stoner L

Wrote review and edited: Keifer A, Bates L, Hanson ED, Horiuchi M, Michos ED, Kucharska-Newton A, Matsushita K, Hughes TM 
Conceptualization, analysis, wrote original draft preparation, wrote review and edited: Lassalle PP Conceptualization, supervision, wrote review and edited: Tanaka $\mathrm{H}$

\section{Availability of data and materials}

Data availability and detailed policies for requesting Atherosclerosis Risk in Communities (ARIC) data can be found at https://sites.cscc.unc.edu/aric/pubs-policies-and-forms-pg. Select ARIC data can also be obtained from the NHLBI BioLINCC repository (https://biolincc.nhlbi.nih.gov/home/).

\section{Financial support and sponsorship}

The Atherosclerosis Risk in Communities study has been funded in whole or in part with Federal funds from the National Heart, Lung, and Blood Institute, National Institutes of Health, Department of Health and Human Services, under Contract nos. (HHSN268201700001I, HHSN268201700002I, HHSN268201700003I, HHSN268201700005I, HHSN268201700004I). The study was supported by R01AG053938. The authors thank the staff and participants of the ARIC study for their important contributions. Heffernan K received support from the Lerner Center for Public Health Promotion, Syracuse University.

\section{Conflicts of interest}

Matsushita K received research funding and personal fee from Fukuda Denshi outside of the work. Other authors declared that there are no conflict of interest.

\section{Ethical approval and consent to participate}

This observational study is reported in accordance with STROBE (STrengthening the Reporting of OBservational studies in Epidemiology) guidelines ${ }^{[15]}$. The study was approved by the Institutional Review Boards at all field centers, coordinating center, and central labs and reading centers.

\section{Consent for publication}

Participants provided written informed consent.

\section{Copyright}

(c) The Author(s) 2022.

\section{REFERENCES}

1. Laurent S, Cockcroft J, Van Bortel L, et al; European Network for Non-invasive Investigation of Large Arteries. Expert consensus document on arterial stiffness: methodological issues and clinical applications. Eur Heart J 2006;27:2588-605. DOI PubMed

2. Townsend RR, Wilkinson IB, Schiffrin EL, et al; American Heart Association Council on Hypertension. Recommendations for improving and standardizing vascular research on arterial stiffness: a scientific statement from the American Heart Association. Hypertension 2015;66:698-722. DOI PubMed PMC

3. Kucharska-Newton AM, Stoner L, Meyer ML. Determinants of vascular age: an epidemiological perspective. Clin Chem 2019;65:10818. DOI PubMed PMC

4. Vlachopoulos C, Aznaouridis K, Stefanadis C. Prediction of cardiovascular events and all-cause mortality with arterial stiffness: a systematic review and meta-analysis. J Am Coll Cardiol 2010;55:1318-27. DOI PubMed

5. Ben-Shlomo Y, Spears M, Boustred C, et al. Aortic pulse wave velocity improves cardiovascular event prediction: an individual participant meta-analysis of prospective observational data from 17,635 subjects. J Am Coll Cardiol 2014;63:636-46. DOI PubMed PMC

6. Fico BG, Gourley DD, Wooten SV, Tanaka H. Heart-thigh cuff pulse wave velocity: a novel nontechnical measure of arterial stiffness. Am J Hypertens 2019;32:1051-3. DOI PubMed

7. Reference Values for Arterial Stiffness' Collaboration. Determinants of pulse wave velocity in healthy people and in the presence of cardiovascular risk factors: 'establishing normal and reference values'. Eur Heart J 2010;31:2338-50. DOI PubMed PMC

8. Heffernan KS, Jae SY, Loprinzi PD. Association between estimated pulse wave velocity and mortality in U.S. adults. J Am Coll Cardiol 2020;75:1862-4. DOI PubMed

9. Heffernan KS, Jae SY, Loprinzi PD. Estimated pulse wave velocity is associated with residual-specific mortality: findings from the National Health and Nutrition Examination Survey. J Hypertens 2021;39:698-702. DOI PubMed 
10. Jae SY, Heffernan KS, Kurl S, Kunutsor SK, Laukkanen JA. Association between estimated pulse wave velocity and the risk of stroke in middle-aged men. Int J Stroke 2021;16:551-5. DOI PubMed

11. Jae SY, Heffernan KS, Park JB, et al. Association between estimated pulse wave velocity and the risk of cardiovascular outcomes in men. Eur J Prev Cardiol 2020. DOI PubMed

12. Greve SV, Blicher MK, Kruger R, et al. Estimated carotid-femoral pulse wave velocity has similar predictive value as measured carotid-femoral pulse wave velocity. J Hypertens 2016;34:1279-89. DOI PubMed

13. Vishram-Nielsen JKK, Laurent S, Nilsson PM, et al; MORGAM Project. Does estimated pulse wave velocity add prognostic information? Hypertension 2020;75:1420-8. DOI PubMed

14. Vlachopoulos C, Terentes-Printzios D, Laurent S, et al. Association of estimated pulse wave velocity with survival: a secondary analysis of SPRINT. JAMA Netw Open 2019;2:e1912831. DOI PubMed PMC

15. von Elm E, Altman DG, Egger M, Pocock SJ, Gøtzsche PC, Vandenbroucke JP; STROBE Initiative. The Strengthening the Reporting of Observational Studies in Epidemiology (STROBE) statement: guidelines for reporting observational studies. J Clin Epidemiol 2008;61:344-9. DOI PubMed

16. The Aric Investigators. The atherosclerosis risk in communit (aric) study: design and objectives. Am J Epidemiol 1989;129:687-702. DOI

17. Cortez-cooper MY, Supak JA, Tanaka H. A new device for automatic measurements of arterial stiffness and ankle-brachial index. Am J Cardiol 2003;91:1519-22. DOI PubMed

18. Meyer ML, Tanaka H, Palta P, et al. Repeatability of central and peripheral pulse wave velocity measures: the Atherosclerosis Risk in Communities (ARIC) study. Am J Hypertens 2016;29:470-5. DOI PubMed PMC

19. Schultz MG, Picone DS, Armstrong MK, et al. The influence of SBP amplification on the accuracy of form-factor-derived mean arterial pressure. J Hypertens 2020;38:1033-9. DOI PubMed

20. Papaioannou TG, Protogerou AD, Vrachatis D, et al. Mean arterial pressure values calculated using seven different methods and their associations with target organ deterioration in a single-center study of 1878 individuals. Hypertens Res 2016;39:640-7. DOI PubMed

21. Papaioannou TG, Oikonomou E, Lazaros G, et al. The influence of resting heart rate on pulse wave velocity measurement is mediated by blood pressure and depends on aortic stiffness levels: insights from the Corinthia study. Physiol Meas 2019;40:055005. DOI PubMed

22. Tan I, Spronck B, Kiat H, et al. Heart rate dependency of large artery stiffness. Hypertension 2016;68:236-42. DOI PubMed

23. Siedel J, Hägele EO, Ziegenhorn J, Wahlefeld AW. Reagent for the enzymatic determination of serum total cholesterol with improved lipolytic efficiency. Clin Chem 1983;29:1075-80. PubMed

24. Friedewald WT, Levy RI, Fredrickson DS. Estimation of the concentration of low-density lipoprotein cholesterol in plasma, without use of the preparative ultracentrifuge. Clin Chem 1972;18:499-502. PubMed

25. Warnick GR, Mayfield C, Benderson J, Chen JS, Albers JJ. HDL cholesterol quantitation by phosphotungstate-Mg ${ }^{2+}$ and by dextran sulfate- $\mathrm{Mn}^{2+}$-polyethylene glycol precipitation, both with enzymic cholesterol assay compared with the lipid research method. Am J Clin Pathol 1982;78:718-23. DOI PubMed

26. Overholser BR, Sowinski KM. Biostatistics primer: part 2. Nutr Clin Pract 2008;23:76-84. DOI PubMed

27. Martin Bland J, Altman D. Statistical methods for assessing agreement between two methods of clinical measurement. Lancet 1986;327:307-10. DOI

28. Wilkinson IB, Mceniery CM, Schillaci G, et al. ARTERY Society guidelines for validation of non-invasive haemodynamic measurement devices: part 1, arterial pulse wave velocity. ARTRES 2010;4:34. DOI

29. Hametner B, Wassertheurer S, Mayer CC, Danninger K, Binder RK, Weber T. Aortic pulse wave velocity predicts cardiovascular events and mortality in patients undergoing coronary angiography: a comparison of invasive measurements and noninvasive estimates. Hypertension 2021;77:571-81. DOI PubMed

30. Stamatelopoulos K, Georgiopoulos G, Baker KF, et al; Pisa COVID-19 Research Group; Newcastle COVID-19 Research Group. Estimated pulse wave velocity improves risk stratification for all-cause mortality in patients with COVID-19. Sci Rep 2021;11:20239. DOI PubMed PMC

31. Lefferts WK, Augustine JA, Spartano NL, Atallah-Yunes NH, Heffernan KS, Gump BB. Racial differences in aortic stiffness in children. J Pediatr 2017;180:62-7. DOI PubMed PMC

32. Heffernan KS, Jae SY, Wilund KR, Woods JA, Fernhall B. Racial differences in central blood pressure and vascular function in young men. Am J Physiol Heart Circ Physiol 2008;295:H2380-7. DOI PubMed

33. Schutte AE, Kruger R, Gafane-Matemane LF, Breet Y, Strauss-Kruger M, Cruickshank JK. Ethnicity and arterial stiffness. Arterioscler Thromb Vasc Biol 2020;40:1044-54. DOI PubMed

34. Paini A, Boutouyrie P, Calvet D, Tropeano AI, Laloux B, Laurent S. Carotid and aortic stiffness: determinants of discrepancies. Hypertension 2006;47:371-6. DOI PubMed

35. Niiranen TJ, Kalesan B, Hamburg NM, Benjamin EJ, Mitchell GF, Vasan RS. Relative contributions of arterial stiffness and hypertension to cardiovascular disease: the Framingham heart study. J Am Heart Assoc 2016;5:e004271. DOI PubMed PMC

36. Rajzer MW, Wojciechowska W, Klocek M, Palka I, Brzozowska-Kiszka M, Kawecka-Jaszcz K. Comparison of aortic pulse wave velocity measured by three techniques: Complior, SphygmoCor and Arteriograph. J Hypertens 2008;26:2001-7. DOI PubMed

37. Wilkinson IB, McEniery CM, Cockcroft JR. Arteriosclerosis and atherosclerosis: guilty by association. Hypertension 2009;54:1213-5. DOI PubMed

38. Cecelja M, Chowienczyk P. Dissociation of aortic pulse wave velocity with risk factors for cardiovascular disease other than hypertension: a systematic review. Hypertension 2009;54:1328-36. DOI PubMed 
39. Wang X, Ye P, Cao R, et al. Triglycerides are a predictive factor for arterial stiffness: a community-based 4.8-year prospective study. Lipids Health Dis 2016;15:97. DOI PubMed PMC

40. Tomiyama H, Hashimoto H, Tanaka H, et al; baPWV/cfPWV Collaboration Group. Synergistic relationship between changes in the pulse wave velocity and changes in the heart rate in middle-aged Japanese adults: a prospective study. J Hypertens 2010;28:687-94. DOI PubMed

41. Stoner L, Meyer ML, Kucharska-Newton A, et al. Associations between carotid-femoral and heart-femoral pulse wave velocity in older adults: the Atherosclerosis Risk in Communities study. J Hypertens 2020;38:1786-93. DOI PubMed PMC

42. Meyer ML, Tanaka H, Palta P, et al. Correlates of segmental pulse wave velocity in older adults: the Atherosclerosis Risk in Communities (ARIC) Study. Am J Hypertens 2016;29:114-22. DOI PubMed PMC

43. Jae SY, Heffernan KS, Kurl S, Kunutsor SK, Laukkanen JA. Association between estimated pulse wave velocity and the risk of heart failure in the Kuopio Ischemic Heart Disease Risk Factor Study. J Card Fail 2021;27:494-6. DOI PubMed

44. Ji C, Gao J, Huang Z, et al. Estimated pulse wave velocity and cardiovascular events in Chinese. Int J Cardiol Hypertens 2020;7:100063. DOI PubMed PMC

45. Hsu PC, Lee WH, Tsai WC, et al. Usefulness of estimated pulse wave velocity in prediction of cardiovascular mortality in patients with acute myocardial infarction. Am J Med Sci 2021;361:479-84. DOI PubMed

46. Hsu PC, Lee WH, Tsai WC, et al. Comparison between estimated and brachial-ankle pulse wave velocity for cardiovascular and overall mortality prediction. J Clin Hypertens (Greenwich) 2021;23:106-13. DOI PubMed

47. Greve SV, Laurent S, Olsen MH. Estimated pulse wave velocity calculated from age and mean arterial blood pressure. Pulse (Basel) 2017;4:175-9. DOI PubMed PMC

48. Liu Y, Sugiyama D, Hirata A, et al. Abstract 11254: double product privilege in predicting all-cause and cardiovascular disease mortality compare to heart rate and blood pressure separately, the Nippon-data 80 study. Circulation 2019;140:A11254. DOI

49. Inoue R, Ohkubo T, Kikuya M, et al. Predictive value for mortality of the double product at rest obtained by home blood pressure measurement: the Ohasama study. Am J Hypertens 2012;25:568-75. DOI PubMed

50. van Hout MJ, Dekkers IA, Lin L, et al. Estimated pulse wave velocity (ePWV) as a potential gatekeeper for MRI-assessed PWV: a linear and deep neural network based approach in 2254 participants of the Netherlands Epidemiology of Obesity study. Int $J$ Cardiovasc Imaging 2021. DOI PubMed 